trends in the statistics of occupational diseases to discover the real trends behind year-to-year fluctuations.

\section{MALIGNANT HEMOPATHIES DUE TO PROFESSIONAL EXPOSURE IN MOROCCO}

S Touil*, D Lahlou, B Benali, A El Kholti. Casablanca Faculty of Medicine and Pharmacy, Hassan II University, Morocco

\subsection{6/oemed-2018-ICOHabstracts. 1106}

Introduction Malignant hemopathies are rare diseases whose professional origin is probably underestimated, despite the growing number of epidemiological studies on this subject. The important role of extraprofessional factors (especially genetic factors), the rarity of malignant hemopathies, their heterogeneity, and their significant onset after carcinogenic exposure, all contribute to explain the difficulties of etiological research in regards of occupational factors.

Methods The aim of this work is to study the various work related malignant hemopathies recognised by the legislator and their etiologies, based on data from the literature as well as the Moroccan occupational diseases charts .

Results Only benzene and ionising radiation are recognised as undisputable carcinogens for blood-forming organs. Thus, different types of leukaemia occurring in the context of occupational exposure to these toxic substances, are included in the occupational diseases charts and are, for this reason, compensable. Nonetheless, there are uncertainties regarding the induction of malignant hemopathies by exposure to certain pesticides, organic solvents, infectious agents and electromagnetic fields for which further epidemiological studies are required.

Discussion Since the only agents known for their induction of malignant hemopathies and are recognised by the Moroccan regulations are benzene and ionising radiation, it is necessary to push the interrogation to establish the causal link to influence the repair of other cancers due to alternate professional exposures and to put in place preventive actions.

Conclusion Prolonged conservation of medical records of the exposed employees and the appeal to the responsible committee are necessary for the improvement of knowledge and the evolution of regulation.

In terms of prevention, medical surveillance, the protection of employees and the use of less toxic alternatives as soon as possible are obviously essential.

\section{THE IMPACT OF METABOLIC SYNDROME ON KAROSHI FROM OVERWORK}

${ }^{1}$ Chung-Ching Wang, 'Wei-Liang Chen, 'Sheng-Ta Chiang, 'Ying-Chuan Wang, 'FangYih Liaw, ${ }^{3}$ Wei-Te Wu, ${ }^{1,2,3}$ Saou-Hsing Liou*. ${ }^{1}$ Division of occupational medicine, Department of Family and Community Medicine, Tri-Service General Hospital, National Defense Medical Centre, Taipei, Taiwan, Republic of China; ${ }^{2}$ Department of Public Health, National Defense Medical Centre, Taipei, Taiwan, Republic of China; ${ }^{3}$ National Institute of Environmental Health Science, National Health Research Institutes, Miaoli, Taiwan, Republic of China

10.1136/oemed-2018-ICOHabstracts.1107

Introduction Cardiovascular and cerebrovascular diseases (CVD) were found to be associated with overwork in Asia countries, as was death from overwork or known as karoshi. Emerging evidences pointed out a strong dose-response association between working long hours and risk of CVD. However, there was little information concerning the effect of metabolic syndrome on CVD mortality in patients with overwork or without overwork. The aim of this study was to investigate the risk of karoshi from overwork among bus drivers with metabolic syndrome (MetS).

Method In the Taiwan Bus Driver Cohort Study during the period 2005-2012, 1014 professional drivers completed comprehensive studies. Working pattern questionnaire, job stress questionnaires, Swedish occupational fatigue inventory, stress satisfaction offset score (SSOS), biochemical measurements, and physical examinations were used to assess the overwork status and the presence of metabolic syndrome. This cohort was linked to the National Health Insurance Research Dataset to determine whether these workers had higher risk of karoshi from overwork.

Results There were 1014 enrolled bus driver with mean age of $41.05 \pm 7.83$. The demographic characteristics, biochemical indices, and job stress scores of drivers were presented. For cardiovascular disease mortality, the unadjusted HRs for participants with MetS were $2.00 \quad(95 \%$ CI: 1.47 to 2.73; $\mathrm{p}<0.001)$ with comparison to those without MetS. After additional adjustment of pertinent variables, positive association remained essentially unchanged $(\mathrm{HR}=1.47,95 \% \mathrm{CI}: 1.04$ to 2.09; $\mathrm{p}=0.030)$. In terms of individual metabolic risk components for cardiovascular disease mortality, BMI, high blood pressure, and high fasting glucose were found to be statistically significant for risk of mortality. After adjusting for covariates, BMI and high blood pressure were two important predictors of CVD mortality.

Conclusion Our study highlighted that the incurrence of metabolic syndrome in bus driver combined with overwork was associated with increased cardiovascular mortality. Regarding metabolic components, BMI and high blood pressure were prognostic predictors of CVD mortality.

\section{IMPORTANCE OF OCCUPATIONAL VISUAL SCREENING: JHAH MODEL}

${ }^{1}$ Sajjid Abbasi, ${ }^{2}$ Moazzam Zaidi ${ }^{*}$, ${ }^{2}$ Mohammed Mohammed, ${ }^{2}$ Marwan Behisi. 'Occupational Optometrist, John Hopkins Aramco Healthcare (JHAH); ${ }^{2}$ Specialist Occupational Medicine, JHAH

\subsection{6/oemed-2018-ICOHabstracts.1108}

Introduction Saudi Aramco is the national oil company of Saudi Arabia. JHAH is the healthcare provider for eligible Saudi Aramco employees and their dependents.

The JHAH vision conservation program provides quality assurance for prescription safety spectacles, visual assessment of cases referred from occupational medicine, eye examination for laser users, visual assessment for drivers and heavy equipment and crane operators, and a glaucoma screening program.

Glaucoma was the cause for the permanent disability of 38 staff members due to blindness between 1975-1984. As a result, Saudi Aramco decided to keep its glaucoma screening program in-house and not outsource it as other large companies do.

Methods Employees above the age of 45 are enrolled in glaucoma screening and are assessed once every 5 years.

During the screening examination, an employee is provided with a detailed history with symptoms, visual acuity assessment, an ocular health examination and intraocular pressure measurements. If a test comes back abnormal, the employee is scheduled for a detailed screening examination in the 